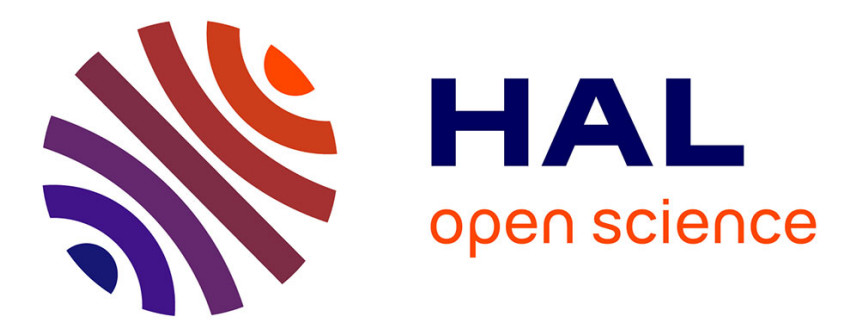

\title{
Poly(4-vinyl pyridine) radiografted PVDF track etched membranes as sensors for monitoring trace mercury in water
}

H Bessbousse, N Zran, J Fauléau, B Godin, V Lemée, T Wade, Marie-Claude Clochard

\section{To cite this version:}

H Bessbousse, N Zran, J Fauléau, B Godin, V Lemée, et al.. Poly(4-vinyl pyridine) radiografted PVDF track etched membranes as sensors for monitoring trace mercury in water. International Journal for Radiation Physics and Chemistry, 2015, in press. 10.1016/j.radphyschem.2015.03.011 . hal-01142483

\section{HAL Id: hal-01142483 https://hal.science/hal-01142483}

Submitted on 15 Apr 2015

HAL is a multi-disciplinary open access archive for the deposit and dissemination of scientific research documents, whether they are published or not. The documents may come from teaching and research institutions in France or abroad, or from public or private research centers.
L'archive ouverte pluridisciplinaire $\mathbf{H A L}$, est destinée au dépôt et à la diffusion de documents scientifiques de niveau recherche, publiés ou non, émanant des établissements d'enseignement et de recherche français ou étrangers, des laboratoires publics ou privés. 


\title{
Poly(4-vinyl pyridine) radiografted PVDF track etched membranes as sensors for monitoring trace mercury in water
}

\author{
H. Bessbousse ${ }^{1}$, N. Zran ${ }^{1}$, J. Fauléau ${ }^{1}$, B. Godin ${ }^{1}$, V. Lemée ${ }^{1}$, T. Wade ${ }^{1^{*}}$, M-C. Clochard ${ }^{1 *}$ \\ 1. Laboratoire des Solides Irradiés, UMR7642, Ecole Polytechnique, 91128 PALAISEAU, France
}

\section{ABSTRACT}

By a radiation-induced grafting technique, we have functionalized track-etched nanoporous polymer membranes with mercury sensitive poly-4-vinyl pyridine (P4VP). Coating of these membranes with a very thin layer of gold results in an electrochemical sensor that is very selective and highly sensitive for mercury LOD $5 \mathrm{ng} / \mathrm{L}$ - well below the norms for water $(0.015 \mu \mathrm{g} / \mathrm{L}$ potable water and $0.5 \mu \mathrm{g} / \mathrm{L}$ residual waters - French water norms of 27 October 2011). E-beam irradiation permitted optimization of the radiografting synthesis on PVDF thin films prior to ion-track grafting. Synthesis and characterization by EPR, FESEM and FTIR are described in detail. A comparison between FTIR in ATR and transmission modes enabled us to localize the grafting on the surface of the e-beam irradiated PVDF films allowing us to extrapolate what happens on the etched tracks.

Using Square Wave Anodic Stripping Voltammetry (SW-ASV), mercury concentrations of $1 \mu \mathrm{g} / \mathrm{L}$ are detected in two hours and low $\mathrm{ng} / \mathrm{l}$ concentrations are detected after 24 hours of adsorption. The adsorption is passive so sensors do not require instrumentation and the analysis takes only 3-4 minutes. Also, the P4VP functionalized sensor appears insensitive to $\mathrm{pH}$ variations (pHs 3 to 9), high salt concentrations (up to $1 \mathrm{~g} / \mathrm{L}$ ) and the presence of other heavy metals in the same solution.

Key words: Track-etched membrane; vinylpyridine; irradiation; radiografting; mercury detection

* corresponding authors: e-mail: Travis.wade@polytechnique.edu tel:+33 1693345 25; email: marie-claude.clochard@polytechnique.edu tel:+33 169334526 


\section{Introduction}

The needs of water monitoring in different environmental contexts, issued from European and national regulations, push more and more towards real-time, on-site or in situ analyses to improve the reliability of monitoring and to have faster information... Tolerable limits are now in the very low $\mu \mathrm{g} / \mathrm{L}$ range. This requires fast, portable, low-cost, environmentally friendly and sensitive instruments which are able to detect and to quantify heavy metal concentrations in different types of water; however, few options are readily available.

Mercury analysis in water is usually performed by atomic absorption spectroscopy (AAS), atomic fluorescence spectroscopy (AFS) or inductively coupled plasma mass spectroscopy (ICP-MS) often with chemical volatilisation (cold vapour) or other pre-concentration steps. One of the particularities of mercury is that it is often analysed separately from other metals. Detection limits are low $\mathrm{ng} / \mathrm{L}$ to sub ng/L; however, these methods are laborious, expensive, time consuming and require a centralised lab with trained personnel.

Most of the state-of-the art technology is not adequate to meet the demands of sensor manufacturers, integrators or end users. Indeed, electrochemical probes, based on anodic stripping voltammetry (ASV), appear cheap, sensitive enough and have a fast response time. It is thus a very promising market. Nevertheless, on the market today, the most sensitive are based on mercury which is very toxic and the cheapest are disposable screen printed electrodes (SPES) which are portable and non- toxic but lack sensitivity.

The present paper validates the technical potential of a 3-D sensor [1] which is able to quantify mercury ions at trace levels, in situ, on-site and/or on-line, using passive sampling. This environmentally friendly sensor relies on ion-track etched nanoporous functionalized membranes made of poly(vinylidene difluoride) (PVDF). The functionalization takes place inside the tracks using radiation induced grafting. The quantitative determination of ionic species is performed via SW-ASV. The multi-ion sensor resulting from poly(acrylic acid) functionalization has already shown good performances for lead in pure water [2] but recent tests performed in natural waters of various 
compositions (salts, $\mathrm{pH}$ ) have demonstrated this sensor has problems with interfering ions, low pHs (<4.5) or complicated matrixes (Fig. 1).

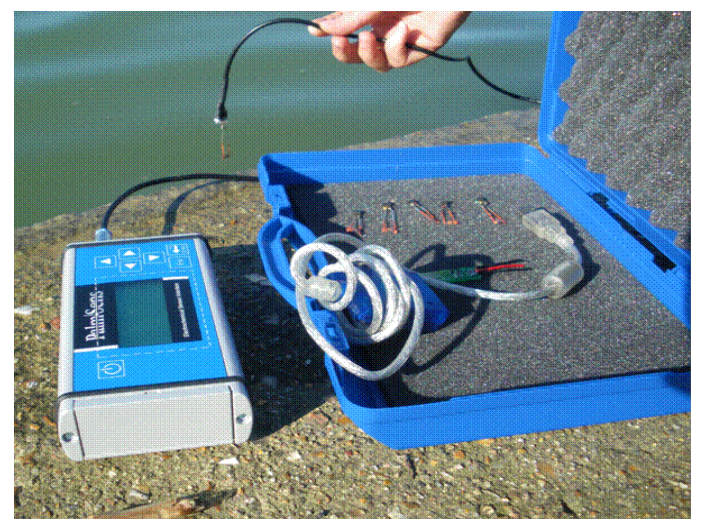

Figure 1. Prototype photograph directly immersed in a natural water.

In light of this, efforts are now focused on specific ions detection permitting this sensor to operate in extreme conditions of $\mathrm{pH}$ and salt interferences. The publication here reports on specific radiation induced functionalization of PVDF track-etched membrane with mercury sensitive poly-4vinyl pyridine (P4VP). Using SW-ASV, we demonstrate the electrochemical sensor's limits of detection, selectivity towards mercury ions among other solvated heavy metals and sensitivity to its environment in a large range of $\mathrm{pH}$ (from 3 to 9) and salt concentrations.

\section{Experimental section}

Swift heavy ions and electron beam irradiations

Swift heavy ion (SHI) irradiation was performed at GANIL in France. Ions of $\mathrm{Kr}^{31+}$ were accelerated by one cyclotron to $10 \mathrm{MeV} / \mathrm{mau}$. SME beam line is equipped with an experimental set-up allowing irradiation of polymer film surfaces of $4 \times 30(\mathrm{~cm} \times \mathrm{cm})$. Required fluences for this work were $10^{9}$ and $10^{10} \mathrm{~cm}^{-2}$.

Electron beam irradiations were performed at the Laboratoire des Solides Irradiés (LSI) in France on SIRIUS equipment. SIRIUS is a recent Van-de-Graaf accelerator of $2.5 \mathrm{MeV}$ electron beam. The dose rate was $100 \mathrm{kGy} \cdot \mathrm{h}^{-1}$. Doses ranged from 1 to $100 \mathrm{kGy}$. 


\section{Chemical etching}

SHI irradiated PVDF films were etched in $\mathrm{KOH}(10 \mathrm{~N}): \mathrm{KMnO}_{4}(0.25 \mathrm{~N})$ solution at $65^{\circ} \mathrm{C}$ for 12 and 15 min. Resulting membranes were successively washed by deionized water, potassium metabisulfite $(15 w t \%)$ and deionized water again.

\section{Radiografting conditions}

E-beam irradiated PVDF films and track-etched PVDF membranes were immersed in a deaerated 4-vinylpyridine solution under nitrogen. The solvent was $\mathrm{N}$-methylpyrrolidone (NMP). Its concentration effect has been investigated from $25 \mathrm{v} / \mathrm{v} \%$ in water to $100 \mathrm{v} / \mathrm{v} \%$. The reaction temperature was set at $65{ }^{\circ} \mathrm{C}$. The grafting time varied depending on the ionizing radiation used. The resulting poly(vinylidene difluoride)-grafted-poly(4-vinylpyridine) (PVDF-g-P4VP) membranes were washed by ethanol in soxhlet apparatus for $16 \mathrm{~h}$. No polymerization inhibitor was added in the monomer solution. Grafting yields were determined gravimetrically. Preparing $\mathrm{KBr}$ pellets with various pyridine content, the grafting degrees were also determined by FTIR after calibration of the pyridinium peak intensity at $1600 \mathrm{~cm}^{-1}$ (Beer-Lambert's law).

\section{Anodic Stripping Voltammetry measurements}

PVDF-g-P4VP track-etched membranes were changed into an electrochemical nanoporous sensors, called CAPTÔT, thanks to the fabrication protocol described in [1,2]. CAPTÔT was then immersed in the aqueous solution to analyse for an adsorption time ranging from 30 minutes to 24 hours. During this step, the mercury solvated ions present in the aqueous solution (if any) were trapped inside the sensor nanopores by complexation with P4VP.

Applying a potential of $-0.800 \mathrm{~V}$, for a deposition time of 100 seconds permits the electrodeposition of the trapped ions on the cathode. The electrochemical analysis was done by SW-ASV scanning the voltage from $-0.800 \mathrm{~V}$ to $+1.200 \mathrm{~V}$ with a step potential of $0.003 \mathrm{~V}$, a frequency of $75 \mathrm{~Hz}$ and an amplitude of $0.030 \mathrm{~V}$. Calibration curves of PVDF-gP4VP were established in order to accurately measure the amount of detected mercury ions by CAPTÔT. 


\section{Results and discussion}

\section{Synthesis of nanoporous mercury-sensitive membranes}

These new membrane electrodes are based on ion-track etched functionalized polymer membranes. The key feature of this system is that the pre-concentration step is realized by passive adsorption inside the functionalized nanopores and not by electro-deposition. This is the most critical step for sensitivity (Fig. 2).

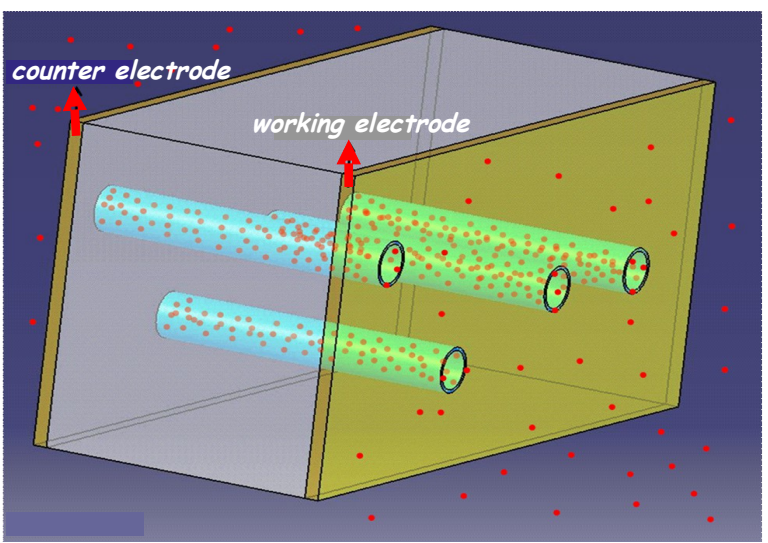

Figure 2. The conception of the sensor in 3D. The red points are heavy-metal ions adsorbing into the nanopores.

The nanopores are created following irradiation of a thin polymer film (PVDF, $10 \mu \mathrm{m}$-thick) by swift heavy ions and subsequent chemical etching of the ion rectilinear tracks through the entire thickness of the polymer film. The chemical etching is done by hydrolysis from polymer chains ends created by chains scission during ion bombardment. A strong alkaline solution ( $\mathrm{KOH} 10 \mathrm{~N}$ ) in synergy with an oxidant such as $\mathrm{KMnO}_{4}$ is needed to reveal the tracks into cylindrical nanopores under our experimental conditions. In PVDF, the residual radicals formed during irradiation all along the tracks are still present in the pore walls after etching when the diameters are less than a few hundred $\mathrm{nm}$ [3]. These radicals trapped in the crystallites of PVDF are able to initiate a polymerization in the presence of a vinyl monomer such as acrylic acid with a maximum efficiency and reproducibility at a diameter of $50 \mathrm{~nm}$ [4]. The functionalization by so called radiation-induced grafting or more commonly radiografting, occurs locally inside the nanopores and takes advantage of radicals presence on these nanopores walls. The functionalization gives the nanoporous membrane complexation properties necessary to trap toxic heavy metal ions solvated in water. The P4VP was chosen for its high affinity for mercury ions $[6,7]$. 


\subsection{Radiation-induced grafting of 4-vinylpyridine on polymer membranes}

4-vinylpyridine (4VP) monomer reactivity towards radiation-induced grafting has been well established on several solid polymers $[8,9]$ but has not yet been stated in the literature for PVDF. Thus, the protocol has to be adapted. Etched tracks are very narrow channels with a diameter of 50 $\mathrm{nm}$ and a length of $10 \mu \mathrm{m}$ (Fig. 3a). The characterization and the localization of the radiografting become difficult when high nanopores densities are reached.

Figure 3. FESEM images of nanoporous track etched membrane cross sections (fluence $10^{10} \mathrm{~cm}^{-2}$ ) a. PVDF; b. PVDF-g-P4VP.

Aa easy way to study the best radiografting conditions with a new vinyl monomer is to first irradiate PVDF films with an electron beam. In PVDF films, the created radicals induced by e-beam irradiation are similar to those created by swift heavy ions. The advantage is that the radicals are homogeneously distributed in the polymer film macroscopically mimicking what happens at the scale of the nanopore wall. It is thus easier to study the radiografting by EPR and FTIR. Once the radiografting protocol has been established using e-beam irradiated PVDF films, it can be directly transferred to ion-track etched nanoporous membranes.

\subsubsection{Radiografted Electron beam irradiated PVDF films with P4VP}

Several e-beam irradiations have been performed at various doses to study the effect on radical formation. The profile of the resulting EPR spectra is typical of alkyl and peroxyl radicals both present in irradiated PVDF films (Fig. 4a) and similar to the EPR spectra obtained for track-etched nanoporous PVDF [4]. The radical concentration logarithmically increases with the dose. 

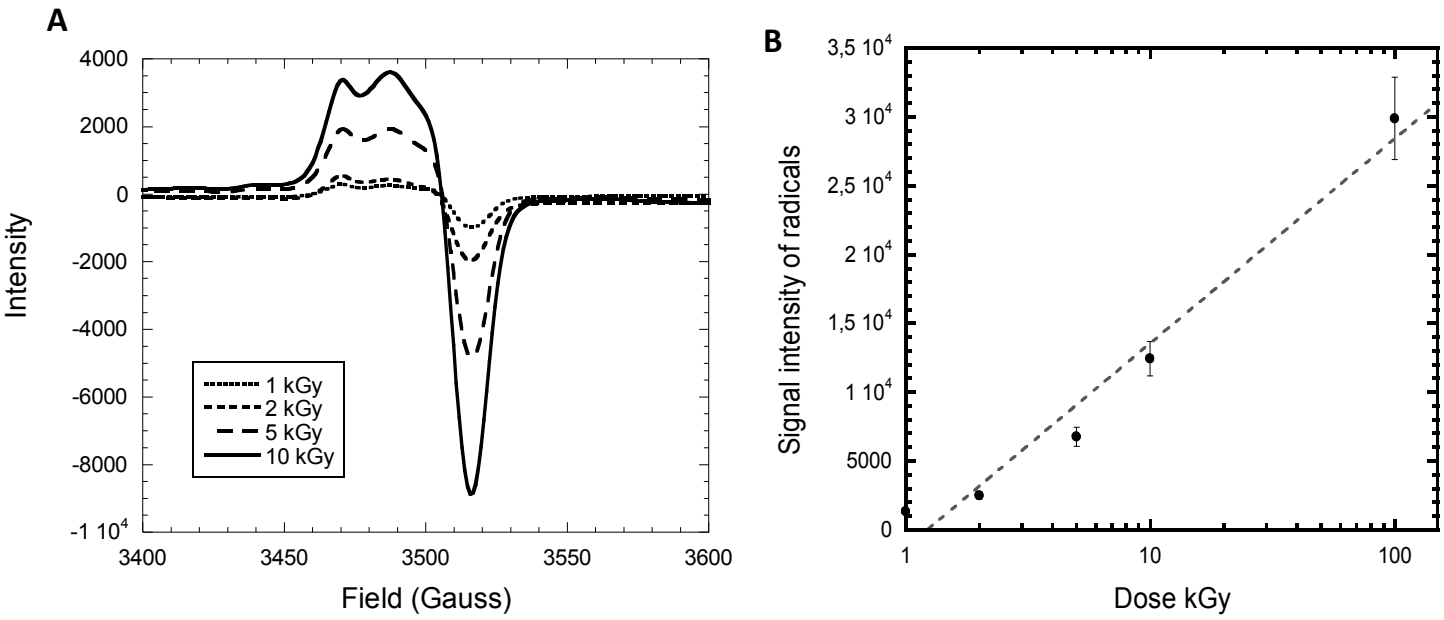

Figure 4. a. EPR spectra of e-beam irradiated PVDF films at various doses; b. Logarithm dependence of radical density versus dose.

These radicals, alkyl and peroxyl, act as initiators for the radical polymerization of 4VP [5]. The radiografting conditions were first performed at a fixed dose of $100 \mathrm{kGy}$ varying the concentration of 4VP monomer in NMP. The films are analyzed by FTIR. In Figure 5, peaks of radiografted P4VP appear clearly at $1600 \mathrm{~cm}^{-1}$ and $1555 \mathrm{~cm}^{-1}$ corresponding to the pyridine ring and in the zone of 3000 $\mathrm{cm}^{-1}$ where $\mathrm{CH}$ vibration bands of PVDF are surrounded progressively by $\mathrm{CH}$ vibration bands of P4VP as the 4VP monomer concentration increases in the radiografting solution. The radiografting yield increases proportionally with the 4VP concentration. 

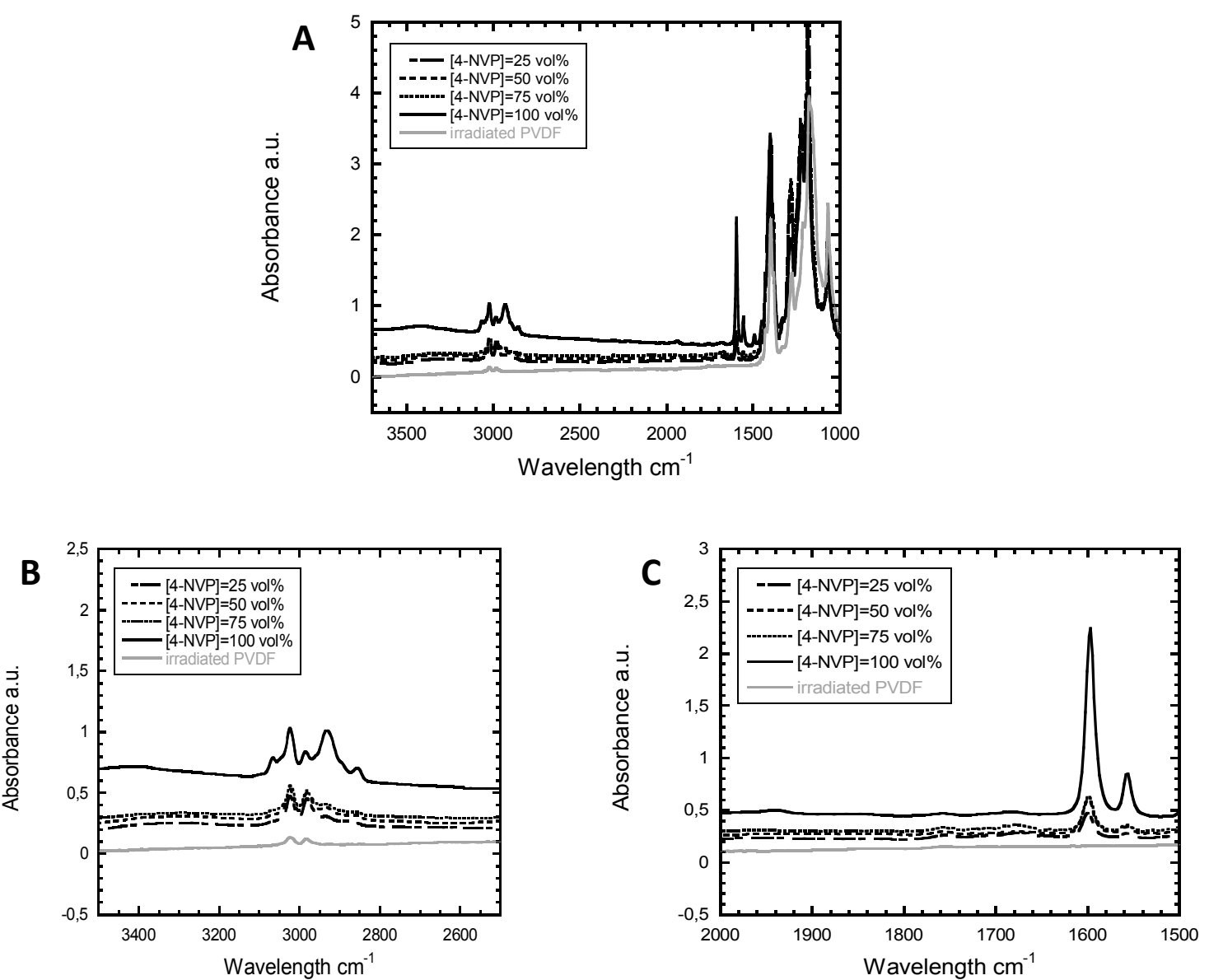

Figure 5. a. Global FTIR spectra registered for PVDF-g-P4VP films after e-beam irradiation (dose of $100 \mathrm{kGy}$; reaction temperature: $65^{\circ} \mathrm{C}$; grafting time : $\left.1 \mathrm{~h}\right) ; b$. zoom of the $3000 \mathrm{~cm}^{-1}$ region; c. zoom between $1500 \mathrm{~cm}^{-1}$ and $2000 \mathrm{~cm}^{-1}$.

For a given dose, pure 4VP monomer solution was found to give the best P4VP radiografting yield on PVDF films. In order to get a suitable system for nanopore walls in further experiments, the best grafting yield obtained from e-beam irradiated PVDF films, which was up to $80 \mathrm{wt} \%$ for $65{ }^{\circ} \mathrm{C}, 100$ kGy and 1 hour reaction time, has to be reduced to 10 wt\% to limit the P4VP chain lengths and not block the nanopores. Our approach was to diminish drastically the reaction time up to 10 minutes and study the effect of doses at $60{ }^{\circ} \mathrm{C}$ and $65{ }^{\circ} \mathrm{C}$. The grafting yield obtained gravimetrically is compared to the dose under these grafting conditions (Fig. 6). A grafting degree of $10 \mathrm{wt} \%$ can be easily achieved in the first doses. A temperature of $60^{\circ} \mathrm{C}$ exhibits a more controlled grafting degree than at $65^{\circ} \mathrm{C}$. 


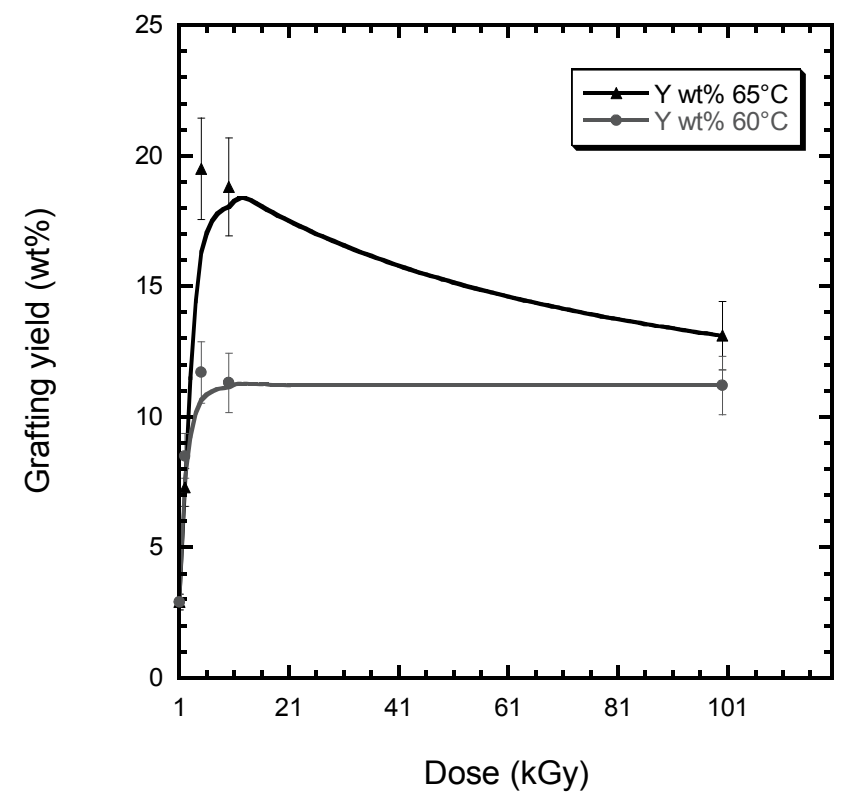

Figure 6. Radiografting yield (wt\%) of PVDF-g-P4VP films in pure 4VP monomer solution for 10 min at $60^{\circ} \mathrm{C}$ and $65^{\circ} \mathrm{C}$ versus the dose (kGy)

Figure 7 displays FTIR spectra in transmission mode. We observe that the radiografted pyridine ring peak at $1600 \mathrm{~cm}^{-1}$ increases up to $5 \mathrm{kGy}$ and reaches a grafting limit with no more increase despite the increasing dose. This means that a dose of $5 \mathrm{kGy}$ is sufficient to reach the maximum radiografting degree of P4VP under the chosen grafting conditions. This grafting protocol suits the conditions for the next step of membrane fabrication by SHI irradiation as fluences of $10^{9} \mathrm{~cm}^{-2}$ and $10^{10} \mathrm{~cm}^{-2}$ correspond to doses in the range of 5 to $10 \mathrm{kGy}$. 


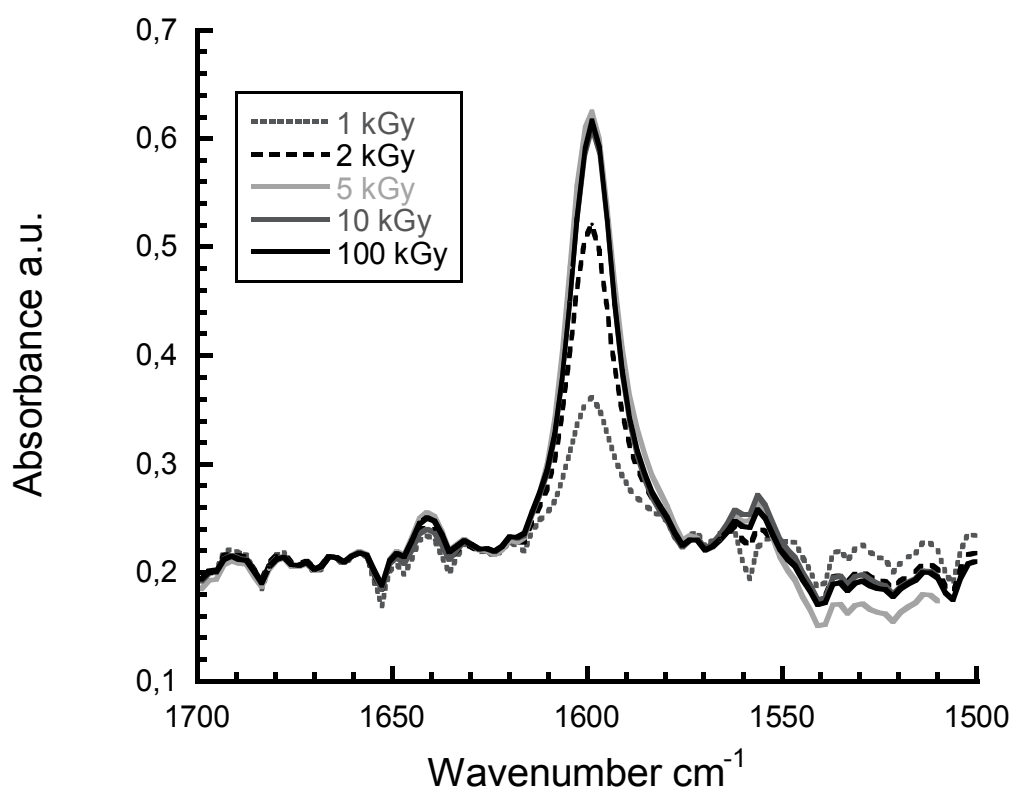

Figure 7. Zoom in the range of $1500 \mathrm{~cm}^{-1}$ and $1700 \mathrm{~cm}^{-1}$ of FTIR spectra in transmission mode of PVDF-g-P4VP films after e-beam irradiation at various doses: 1 kGy, 2 kGy, 5 kGy 10 kGy and 100 kGy. Grafting conditions: 100 vol\% of $4 \mathrm{VP}, 10 \mathrm{~min}, 60^{\circ} \mathrm{C}$.

\subsubsection{Grafting localization}

Two FTIR modes have been used: Attenuated Total Reflexion (ATR) and transmission. The ATR mode proves the first micrometer of the radiografted PVDF films. This mode, based on evanescent FTIR light, gives information of the film surfaces while in the transmission mode, the laser crosses through all the film thickness giving FTIR response of all the material.

FTIR spectra in ATR mode exhibit the pyridine peaks at $1650 \mathrm{~cm}^{-1}, 1600 \mathrm{~cm}^{-1}$ and $1555 \mathrm{~cm}^{-1}$ (Fig. 8a). The most intense peak corresponds to the pyridine ring vibration band at $1600 \mathrm{~cm}^{-1}$, this band is proportional to the logarithm of the irradiation dose. This dependence is the same whatever the reaction temperature. A higher temperature leads to higher 4VP polymerization on the PVDF film surface.

The quaternization reaction by the introduction of a positive charge to form a pyridinyl ring in the polymer structure may explain the peak at $1650 \mathrm{~cm}^{-1}$. [11] Additionally, the intensity of the band at about $1555 \mathrm{~cm}^{-1}$ corresponds to positively charged nitrogen atoms ( Fig. 8a). 

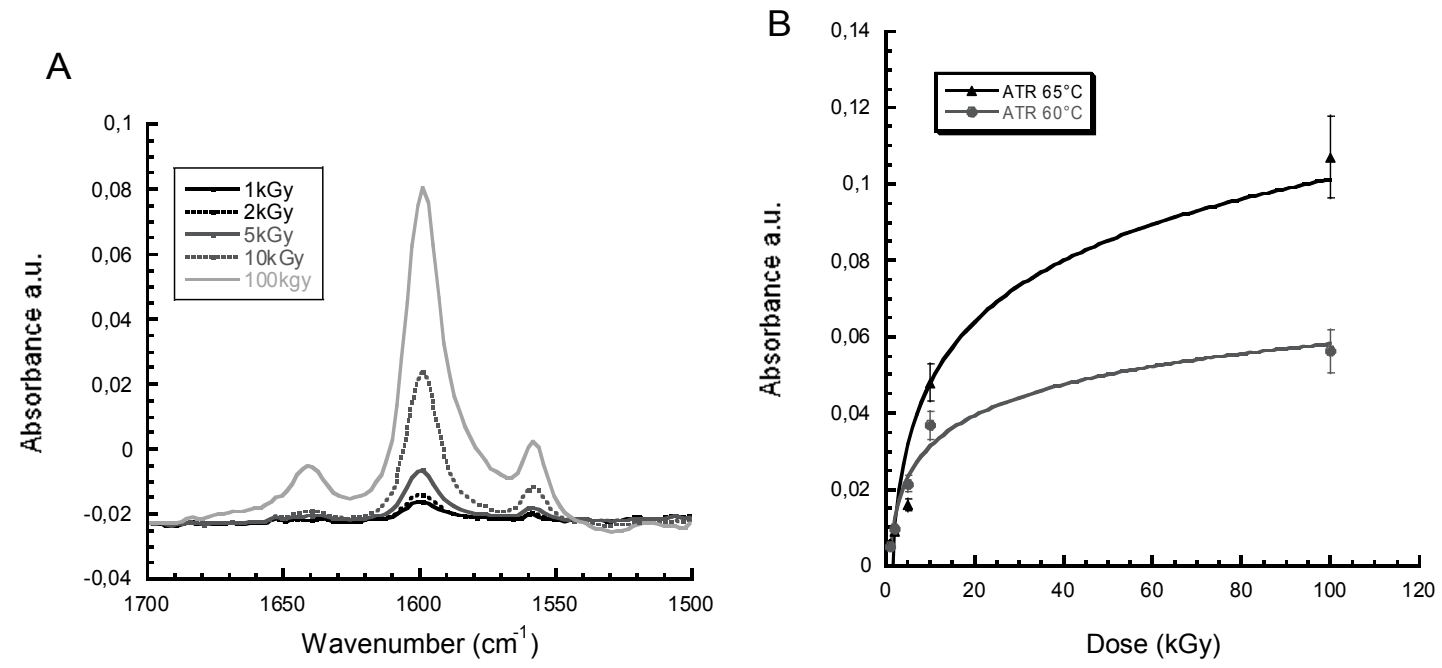

Figure 8: a. Zoom in the range of $1500 \mathrm{~cm}^{-1}$ and $1700 \mathrm{~cm}^{-1}$ of FTIR spectra in ATR mode of PVDF-gP4VP films after e-beam irradiation at various doses: 1 kGy, 2 kGy, 5 kGy 10 kGy and 100 kGy. b. Effect of reaction temperature on the pyridine peaks absorbance at $1600 \mathrm{~cm}^{-1}$. Grafting conditions: 100 vol\% of $4 \mathrm{VP}, 10 \mathrm{~min}, 60^{\circ} \mathrm{C}$.

By comparison to transmission FTIR, the spectra of PVDF-g-P4VP in ATR mode show a continuous increase in the pyridine ring quantity with the low dose range onto the grafted films surface. These FTIR results demonstrate that the grafting occurs mainly on the surface of the film and does not expand inside the films.

After a cryofracture of a PVDF-g-P4VP membrane and doping of the pyridinium cycle by copper ions, an energy dispersive X-ray (EDX) probe coupled to SEM confirms the major location of P4VP on the membrane surface (Fig. 9).

Figure 9: Scan line EDX profile of $\mathrm{Cu}(K$ line) and $F$ ( $K$ line) along the cross-section of a Cu doped ebeam radiografted PVDF-g-P4VP membrane (PVDF initial thickness of $10 \mu \mathrm{m}$, dose : $100 \mathrm{kGy}$ ). Grafting conditions: 100 vol\% of $4 \mathrm{VP}, 10 \mathrm{~min}, 60^{\circ} \mathrm{C}$.

\subsubsection{Etched ion track grafting}


Once the radiografting conditions have been established at the PVDF film surface by e-beam irradiation, the radiografting procotol is directly applied to PVDF track-etched membranes.

In order to get $50 \mathrm{~nm}$ diameter for each track, the tracks are revealed by a chemical attack using $\mathrm{KOH} / \mathrm{KMnO}_{4}$ solution. The radical fraction present on the pore walls after etching depends on the track diameter as their density decreases as $1 / R^{2}$ ( $R$ the etched track radius) from the initial track core [4]. As previously described [3,4], the diameter is proportional to the etching time. However the linear relationship also depends on the fluence. For example, with a fluence of $5.10^{8}$ ions. $\mathrm{cm}^{-2}$, $\mathrm{R}=0.8 \mathrm{x} t$, where $\mathrm{R}$ is the radius in $\mathrm{nm}$ and $t$ the time in min. However, with a fluence of $10^{10}$ ions. $\mathrm{cm}^{-2}$, the relationship changes to $\mathrm{R}=1.6 \mathrm{xt}$.

The etching time is an important parameter as it determines the radical density on the pore walls. As already mentioned, not all vinyl monomers have the same reactivity towards radiografting and some of them need a higher quantity of radicals to start the polymerization. In figure 10 , an etching time of 15 min has found to be insufficient for the polymerization initiation of 4VP in contrast to $12 \mathrm{~min}$. For $12 \mathrm{~min}$ etching time at $65^{\circ} \mathrm{C}$ and selected grafting conditions (100 vol\% of $4 \mathrm{VP}, 10 \mathrm{~min}$, $60^{\circ} \mathrm{C}$ ) the grafting degree was found equal to $Y=6.25 \%$.

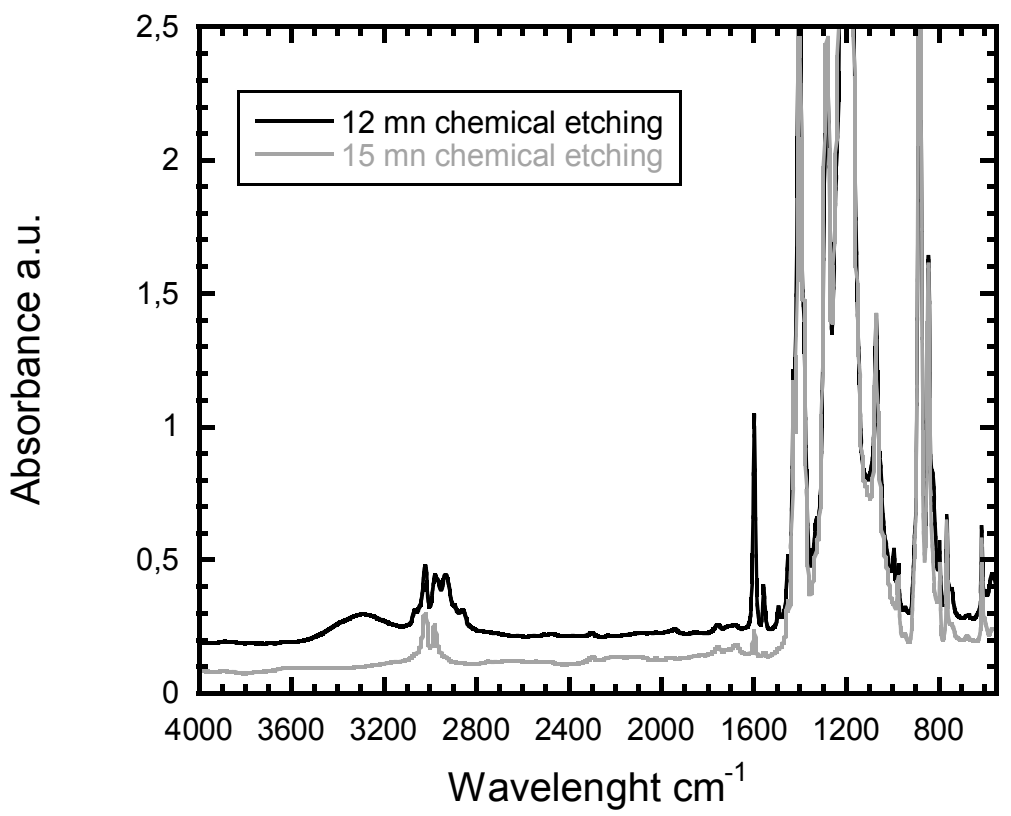

Figure 10 : Functionalized PVDF-g-P4VP nanoporous membranes after $15 \mathrm{~min}$ (grey) and $12 \mathrm{~min}$ (black) of etching time 


\section{Electrochemical detection}

After gold sputtering, the functionalized track-etched membranes will be used as electrodes for SWASV analysis [1,2]. SW-ASV measurements were performed using $\mathrm{Hg}$ standards and $1 \mathrm{mM} \mathrm{NaCl} / \mathrm{HNO}_{3}$ electrolyte. A well-defined $\mathrm{Hg}$ oxidation signal is seen at $+0.500 \mathrm{~V}$ (Fig. 11a). The peak current increases with adsorption time (Fig. 11b).
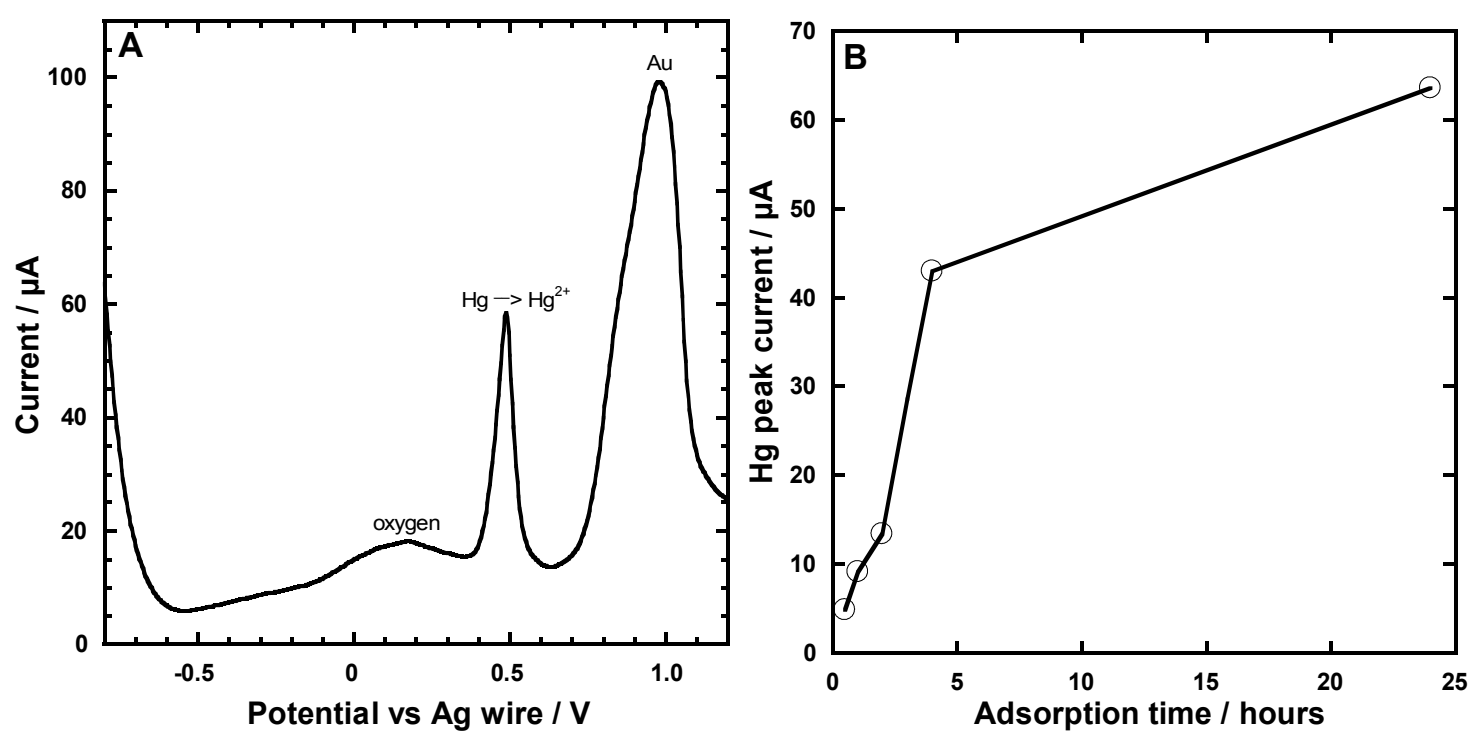

Figure 11 a. SW-ASV analysis curve of $10 \mathrm{ppb} \mathrm{Hg}^{2+}$ standard solution after an adsorption time of 4

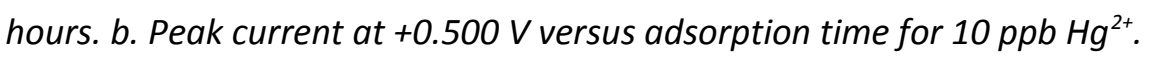

The imposed limit for $\mathrm{Hg}$ in water is $0.015 \mathrm{ppb}$. In order to evaluate the quantitative limits of the sensor at very low concentrations, long adsorption times are required, so P4VP membrane electrodes were placed in a range of standard $\mathrm{Hg}^{2+}$ solutions with concentrations of 0.005 to 0.100 ppb for 24 hours. The P4VP membrane electrodes are found to be very sensitive with a limit of detection (LOD) of $0.005 \mathrm{ppb}$ (Fig. 12). 


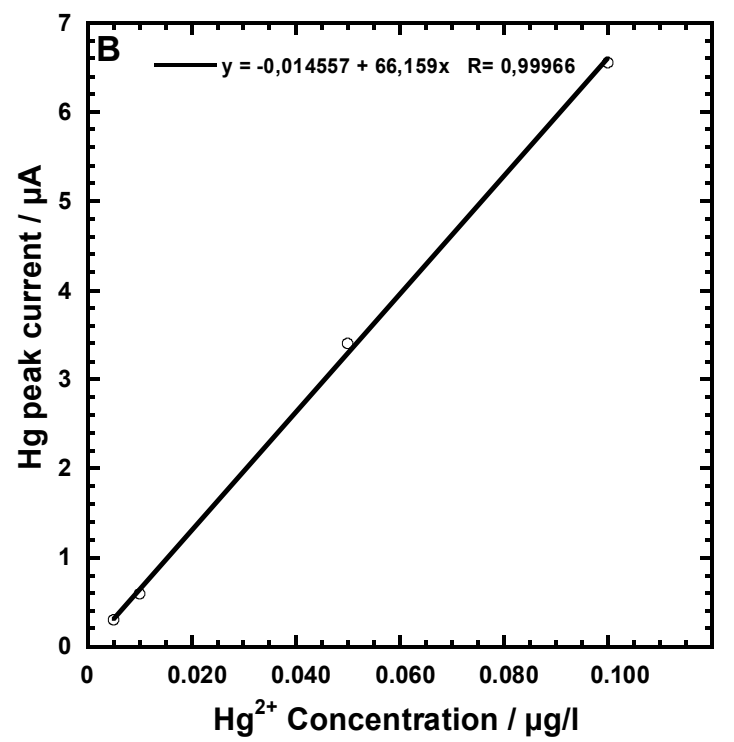

Figure 12: Calibration plot of peak currents at $+0.500 \mathrm{~V}$ versus $\mathrm{Hg}^{2+}$ concentration for the $\mathrm{SW}$ ASV curves in a $10 \mathrm{mM} \mathrm{NaCl} 10 \mathrm{mM} \mathrm{HNO}_{3}$ electrolyte solution.

Next, we evaluated interferences using SW-ASV measurements. Experiments were performed in various conditions (Fig. 13). The results show that the sensors were slightly sensitive to basic pHs (Fig. 13a). The observed shift of the redox potential of $0.05 \mathrm{~V}$ at $\mathrm{pH} 9$ is due to the silver wire pseudoreference electrode as the peak shape was kept intact. In Fig. 13b, salt concentrations were found to have no real significant impact on the SW-ASV measurements $(\Delta V<0.05 \mathrm{~V})$ except for an increase in background current. The sensors have been put in contact with various solvated ions such as $\mathrm{Cu}(\mathrm{II})$, $\mathrm{Zn}(\mathrm{II}), \mathrm{Ni}(\mathrm{II}), \mathrm{Cd}(\mathrm{II})$ and Fe(II) (Fig. 13c). No effect was observed except in the presence of Fe(II) ions. $\mathrm{Fe}(\mathrm{II})$ ions are known to complex $\mathrm{Hg}$ (II) ions [13]. This complexation may explain the decrease of the $\mathrm{Hg}(\mathrm{II})$ trapping by the 4VP functions of the sensors. 


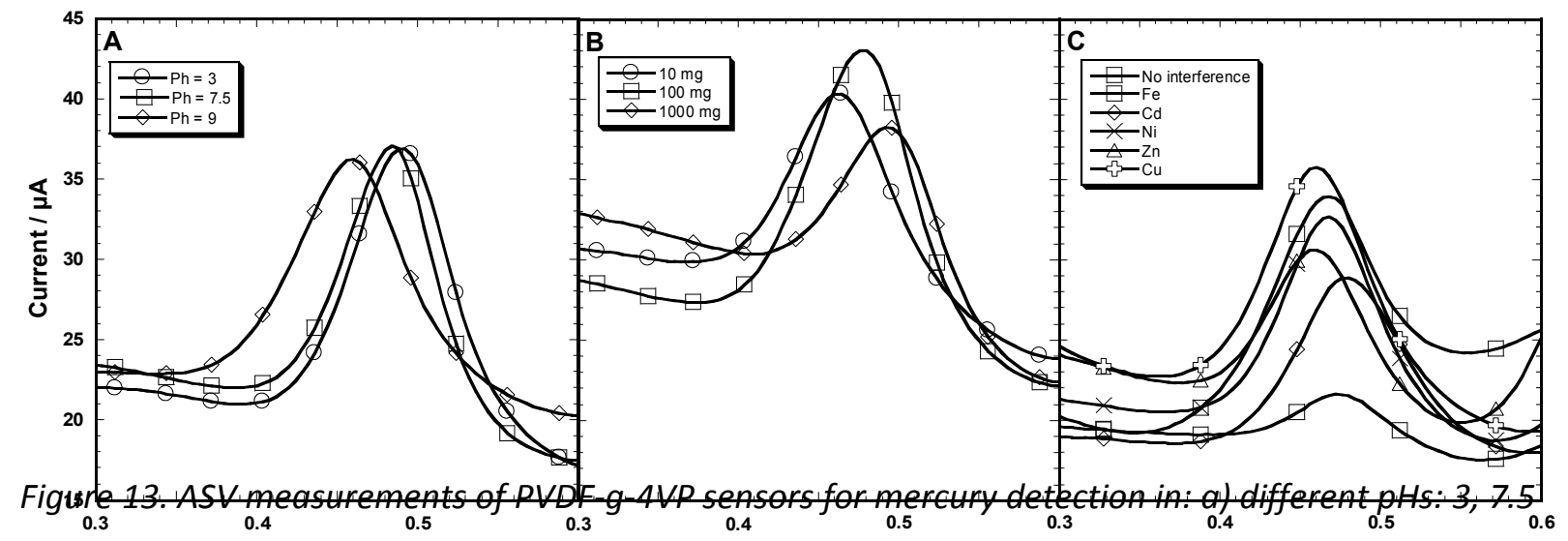

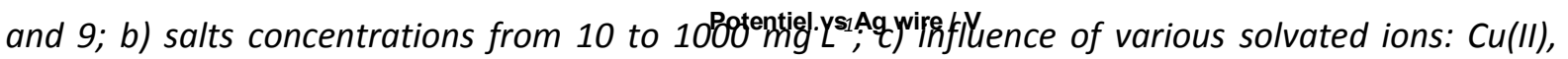
$\mathrm{Zn}(I I), \mathrm{Ni}(I I), \mathrm{Cd}(\mathrm{II})$ and $\mathrm{Fe}(\mathrm{II})$.

\section{Conclusions}

PVDF-g-P4VP nanoporous membranes have been successfully obtained with open nanopores. The radiografting protocol for a fine coverage of P4VP on the PVDF film surface has first been established on e-beam irradiated PVDF films. The FTIR characterization have demonstrated that, in pure 4VP monomer solution, the radiografting occurs on the e-beam irradiated PVDF films surfaces and no diffusion of the 4VP inside the PVDF bulk has been shown. The latter radiografting condition was then applied to freshly track-etched PVDF nanoporous membranes as they are known from previous work to have remaining radicals located on the nano-track walls. Such functionalized PVDF-g-P4VP nanoporous membranes are changed into electrodes and are used as SW-ASV sensors for $\mathrm{Hg}$ detection. The biocompatibility, low-cost, ease-of-use and in situ sampling of the sensor mean that it can be liberally deployed for continuous monitoring of water pollution and quality evolution and for first alert of pollution events. An adsorption time of at least 2 hours is not a handicap since many sensors can be simultaneously immersed and because the adsorption step is passive with an analysis time for each electrode of less than 5 minutes.

\section{Acknowledgements}

The authors want to thank the following french institutions for their financial support: RTRA 2009042T-ASSENSOR, OSEO contract $n^{\circ} \mathrm{A} 1006069 \mathrm{Q}$, and $\mathrm{CEA}^{2}$ TECHNOSANTE transversal Program (acronym ECOSISTEM). The authors also express their warm thanks to the SIRIUS team of LSI and to the CIMAP team at GANIL for their strong implication in radiation experiments. 


\section{References}

[1] T. Wade, M-C Clochard "Method and device using nanoporous membrane for detecting and quantifying heavy metal ions in a fluid" CEA-X-CNRS European patent EP08305237 ; T. Wade, N. Mezailles, H. Bessbousse, M-C Clochard, "nanosensor for capturing charged molecules by using in situ stripping voltammetry" CEA-X-CNRS European patent EP11305112

[2] H. Bessbousse, I. Nandhakumar, M. Decker, M. Barsbay, O. Cuscito, D. Lairez, M-C. Clochard, T.L. Wade, Analytical Methods, 3 (2011) 1351-1359.

[3]Cuscito, O. ; Clochard, M. C. ; Esnouf, S. ; Betz, N. ; Lairez, D. Nuclear Instruments and Methods In Physics Research Section B-Beam Interactions With Materials And Atoms 2007, 265, 309-313

[4] M. Barsbay, O. Güven, H. Bessbousse, T. L. Wade, M.-C. Clochard "Nanopore size tuning of polymeric membranes using the RAFT-mediated radical polymerization" journal of Membrane Science" 445 (2013) 135-145

[5] Guangqun Zhai, Lei Ying, E. T. Kang,K. G. Neoh “Poly(vinylidene fluoride) with grafted 4vinylpyridine polymer side chains for $\mathrm{pH}$-sensitive microfiltration membranes » J. Mater. Chem. 12 (2002) 3508-3515

[6] Bessbousse, Haad; Rhlalou, Thouria; Verchere, Jean-Francois; Lebrun, Laurent « Novel MetalComplexing Membrane Containing Poly(4-vinylpyridine) for Removal of $\mathrm{Hg}$ (II) from Aqueous Solution" J. of Phys. Chem. B 113 (2009) 25, 8588-8598

[7] Bessbousse, Haad; Verchere, Jean-Francois; Lebrun, Laurent "Increase in permeate flux by porosity enhancement of a sorptive UF membrane designed for the removal of mercury(II)" J. of Mem. Sci. 364 (2010) 1-2, 167-176

[8] Kostov, GK; Turmanova, SC « Radiation-initiated graft copolymerization of 4-vinylpyridine onto polyethylene and polytetrafluoroethylene films and anion-exchange membranes therefrom" $\mathrm{J}$. Appl. Polym. Sci 64 (1997) 8, 1469-1475

[9] Burillo, Guillermina. "Temperature and pH-Sensitive Swelling Behavior of Binary DMAEMA/4VP Grafts on Poly(propylene) Films" Macromolecular Materials And Engineering 292 (2007) 2, 214-219

[10] Sanli, Li; Gursel, S.A. "Synthesis and Characterization of Novel Graft Copolymers by RadiationInduced grafting" J. Appl. Polym. Sci. 120 (2011) 2313-2323

[11] Caruso, U.; Maria, D.A.; Panunzi, B.; Roviello, A. "Poly (4-vinylpyridine) as the host ligand of metal-containing chromophores for second-order nonlinear optical active materials " J. Polym. Sci. Part A: Polym. Chem. 40 (2002) 2987-2993. 
[12] A. Hagège et A.Boos, "Analyse de traces et d'ultra-traces d'éléments", Techniques de I'Ingénieur (2013) 3860. 